\title{
Alzheimer Disease and Oxidative Stress
}

\author{
George Perry, Adam D. Cash, and Mark A. Smith* \\ Institute of Pathology, Case Western Reserve University, Cleveland, OH 44106, USA
}

Received 11 February 2002; accepted 18 March 2002

\begin{abstract}
Research in Alzheimer disease has recently demonstrated compelling evidence on the importance of oxidative processes in its pathogenesis. Cellular changes show that oxidative stress is an event that precedes the appearance of the hallmark pathologies of the disease, neurofibrillary tangles, and senile plaques. While it is still unclear what the initial source of the oxidative stress is in Alzheimer disease, it is likely that the process is highly dependent on redox-active transition metals such as iron and copper. Further investigation into the role that oxidative stress mechanisms seem to play in the pathogenesis of Alzheimer disease may lead to novel clinical interventions.
\end{abstract}

\section{INTRODUCTION}

Much of the research performed on Alzheimer disease (AD) over the past decade has focused on oxidative stress mechanisms and its importance in disease pathogenesis. Multiple lines of evidence have implicated oxidative stress and free radical damage to the pathogenesis and possible etiology of AD.

The net effect of oxygen radicals is damage. Such damage found in $\mathrm{AD}$ includes advanced glycation end products [1], nitration [2], lipid peroxidation adduction products $[3,4]$ as well as carbonyl-modified neurofilament protein and free carbonyls $[5,6]$. Importantly, this damage involves all neurons vulnerable to death in $\mathrm{AD}$, not just those containing neurofibrillary tangles. In fact, the spatio-temporal distribution of specific types of damage elegantly reflects the biology and chemistry of each modification.

The cytopathological significance of oxidative damage is seen by the up-regulation of antioxidant enzymes. Heme oxygenase-1 (HO-1) is among the most sensitive and selective indicators of the cellular oxidative stress response and, in $\mathrm{AD}$, we demonstrated that both $\mathrm{HO}-1$ protein and its mRNA are increased in brains of $\mathrm{AD}$ patients $[7,8]$. In later studies, we demonstrated that increased HO-1 expression colocalizes with the altered form of tau $(\tau)$ [9].

\section{OXIDATIVE STRESS AND NFT MODIFICATION}

There is much substantive evidence for progressive oxidative modification of proteins as a consequence of aging. However, the most crucial aspects of the cellular oxidative damage in $\mathrm{AD}$ pathogenesis appear to be the cytoskeletal modifications in neurons susceptible to $\mathrm{AD}$, which in turn play a key role in the irreversible cellular dysfunction that ultimately leads to neuronal death [10]. The most obvious of these cytoskeletal abnormalities are the NFT, which are arguably the most important aspect of the degenerating phenotype in AD-affected neurons. These inclusions largely consist of modified medium and high molecular weight neurofilaments (NFM and NFH, respectively) as well as the microtubule-stabilizing protein $\tau$. In NFT, these two families of protein have an abnormally high level of phosphorylation, which may be explained by their conserved kinase consensus sequences [11]. In addition to hyperphosphorylation, proteins in both NFT and senile plaques have been shown to be modified by the addition of advanced glycation endproducts (AGE) [1] and hydroxynonenal [4].

Some of the antibodies used to detect NFT specifically recognize oxidation-dependent epitopes. We have demonstrated that two antibodies to NFT recognize carbonylmodified epitopes of high molecular weight neurofilaments (NFH) [6]. Although both of these antibodies recognized neurofilaments of the axon in aldehyde-fixed sections, they demonstrated no reactivity in immunoblots with normal axonal protein prepared from tissue unexposed to fixatives. In contrast, if these immunoblots were carbonyl-modified, both antibodies recognized NFH. Significantly, in samples from AD brains, the antibodies recognized NFT epitopes in both tissue sections and NFT-derived NFH in immunoblots without need of carbonyl modification. Sporadic lesionrelated immunoreactivity was seen in control patients. Furthermore, carbonyl-modified NFTs could be isolated from homogenates of AD brain tissue but not from control tissue. Taken together, these results suggest that oxidized NFH is a prominent antigen in NFT [6].

The oxidative conversion of protein side-chains to reactive carbonyl or acylating moieties, or adduction of bifunctional sugar- or lipid-derived products can result in abnormal protein cross-linking. Both neurofilaments and $\tau$ can form noncovalent aggregates in the absence of oxidation. However, they have a limited lifetime, and the proteinderived reactive moieties will preferentially react with solvent 
or other molecules if no suitably reactive and/or positioned group is nearby. We hypothesize that the pathogenic role of oxidative stress, involving direct oxidation and/or modification by oxidation, may be primarily in the covalent "cementing" of reversible associations normally reflecting simple noncovalent affinities. It has been found that many proteins associated with NFT are apparently covalently cross-linked to the filaments since they cannot be removed by denaturants. This is consistent with other studies, which show that oxidative covalent cross-linking of proteins occur predominantly when the proteins are preassociated. The in vitro enzymatic cross-linking of tau could be produced only when the protein substrates were properly associated. Since oxidation-based cross-linking reduces susceptibility to proteolytic degradation by providing nonpeptide bonds [12], covalent crosslinking may in fact represent a neurotoxic event by inhibiting turnover. Simultaneously, several oxidative modifications may generate chemical species, which themselves support further oxidative stress through "redox cycling" or transition metal sequestration.

\section{HOW OXIDATIVE DAMAGE COMPARES TEMPORALLY TO LESIONS OF ALZHEIMER DISEASE}

Closer examination of the spatio-temporal relationship between the presence of oxidative modification and the hallmark $\mathrm{AD}$ lesions reveals an apparent paradox. It has been found that while stable glycation products are predominantly associated with NFT and amyloid- $\beta(\mathrm{A} \beta)$ deposits [1], reversible or rapidly degraded adduction products are primarily in the cytoplasm of vulnerable neurons. This data means that oxidative damage is not restricted to long-lived lesions. In fact, when damage to short-lived molecules is examined, the damage is restricted to cytosolic compartments with NFT and $\mathrm{A} \beta$ being inversely correlated.

To address where reactive oxygen species are produced, we focused our efforts to finding a marker that results from primary attack, rather than more complex secondary reactions, and which involves damage to a cell constituent with a short half-life. Proteins fail in the latter aspect of these criteria because cross-link modifications slow their turnover. Therefore, modifications associated with cross-linking of proteins, while useful to assess history, may reveal less of the current state. However, 8-hydroxyguanosine (8OHG), a nucleic acid modification predominantly derived from hydroxide $(\bullet \mathrm{OH})$ attack of guanidine, is amplified in cytoplasmic RNA in vulnerable neuronal populations [13]. Notably, cases of AD with the most extensive $\mathrm{A} \beta$ deposits show the lowest $8 \mathrm{OHG}$ levels. These findings seem markedly contradictory if we consider $\mathrm{A} \beta$ to be the toxic agent in $\mathrm{AD}$, which is likewise proposed to be a major source of oxidative free radicals [14]. However, neurons containing NFT also have extremely low levels of $8 \mathrm{OHG}$ (ie, current oxidative stress status) despite an obvious history of oxidative damage (ie, advanced glycation endproducts or lipid peroxidation). This suggests that both $\mathrm{A} \beta$ and NFT may serve an antioxidant function, and thus be cellular compensations for increased oxidative stress. The hypothesis that $\mathrm{A} \beta$ plays an antioxidant function is supported by a study of Down syndrome patients, a disease where $\mathrm{A} \beta$ deposits begin in the late teens and in which oxidative stress has been implicated [15].

\section{MITOCHONDRIAL ABNORMALITIES: THE MAIN SOURCE OF OXIDATIVE IMBALANCE?}

Mitochondria are a potential major source of oxidative radicals and oxidative precursors, in the form of $\mathrm{O}_{2}^{-}$ and $\mathrm{H}_{2} \mathrm{O}_{2}$, respectively, since their production is linked to metabolism. In early studies, we found increases in mitochondrial DNA in the cell soma of AD susceptible neurons, which in itself might cause increased oxidative potential. Perhaps more importantly, in situ hybridization studies with a chimeric cDNA probe to a common mitochondrial mutation (5 kilobase $(\mathrm{kb})$ common deletion) have shown at least a 3fold increase in $\mathrm{AD}$ cases compared to controls. Ultrastructural localization of mtDNA with colloidal gold shows that deleted mtDNA is mainly found in abnormal mitochondria (ie, those lacking cristae, swollen and in many cases fused with lipofuscin). These findings suggest that the mtDNA in situ hybridization detected mtDNA proliferation, deletion, and duplication in abnormal mitochondria, many of which have been fused with lysosomes, indicating that they are being turned over.

Quantitative analysis of the colocalization of the mtDNA deletion and $8 \mathrm{OHG}$ in $\mathrm{AD}$ cases demonstrate a strong positive correlation $(r=0.934)$. However, mitochondrial DNA, even that containing the $5 \mathrm{~kb}$ deletion, is relatively spared from oxidative damage, like the formation of $8 \mathrm{OHG}$, in comparison to cytoplasmic nucleic acid (ie, RNA). We therefore suspect that mitochondrial abnormalities correlate with, but do not directly cause, reactive oxygen species (ROS). This may be due to the fact that hydroxide radicals, which are responsible for the formation of $8 \mathrm{OHG}$, have a sphere of diffusion of only $2 \mathrm{~nm}$ and are fairly short lived. Therefore, since damage is topographically distinct, it is likely that $\mathrm{OH}$ radical formation occurs in the cytoplasm rather than the mitochondria and that they are unable to diffuse through the mitochondrial membrane to affect mtDNA. However, abnormal mitochondria may produce excess $\mathrm{H}_{2} \mathrm{O}_{2}$ through the conversion of $\mathrm{O}_{2}^{-}$by mitochondrial superoxide dismutase (SOD). Such $\mathrm{H}_{2} \mathrm{O}_{2}$ is readily diffusible and relatively stable, that is, until interacting with redox-active transition metals where the Fenton reaction produces hydroxyl radicals.

\section{REDOX-ACTIVE METALS IN ALZHEIMER DISEASE: PROXIMAL MARKERS OF OXIDATIVE STRESS?}

Several studies have implicated imbalances of trace elements, including $\mathrm{Al}, \mathrm{Si}, \mathrm{Pb}, \mathrm{Hg}, \mathrm{Zn}, \mathrm{Cu}$, and $\mathrm{Fe}$ in $\mathrm{AD}$. A disruption in the homeostasis of the latter two redox-active metals is particularly significant in light of the increases in oxidative stress parameters, such as lipid peroxidation, and the oxidative damage to NFTs, senile plaques $[1,2,4]$, and nucleic acids [13]. 
Micro particle-induced $\mathrm{x}$-ray emission, recently found that $\mathrm{Zn}(\mathrm{II}), \mathrm{Fe}(\mathrm{III})$, and $\mathrm{Cu}(\mathrm{II})$ are significantly increased in $\mathrm{AD}$ neuropil and that these metals are more concentrated within the core and periphery of senile plaques [16]. These results support earlier studies reporting increased levels of iron, transferrin, and ferritin in AD. An in situ iron detection method revealed a significant association of redox-active iron with both NFT and senile plaques (SP) in AD. The association of iron with NFT may be partly related to the binding of iron to $\tau$, their primary protein constituent [17].

Recent histochemical studies have demonstrated that the direct detection of redox activity in $\mathrm{AD}$ lesions is inhibited by prior exposure of tissue sections to copper and iron selective chelators [4]. Re-exposure of the chelator-treated sections to either copper or iron salts can reinstate activity, suggesting that redox imbalance in $\mathrm{AD}$ is dependent on these metals. It is therefore probable that accumulation of iron and copper is a major source of the production of ROS, which are in turn responsible for the more global oxidative stress parameters observed in $\mathrm{AD}$.

Free iron has been implicated in undergoing redox transitions in vivo with the consequent generation of oxygen free radicals more than any other transition metal. Abnormally high levels of iron and oxidative stress have been shown in a number of neurodegenerative disorders including AD [18], Parkinson disease (PD), multiple system atrophy, and progressive supranuclear palsy. However, oxidative stress is usually associated with increased free iron. Therefore, experimental findings of increased total iron do not necessarily implicate increased oxidative stress if there are concomitant increases in proteins that store iron in redox inert forms. For example, ferritin (which can be over-expressed in PD and $\mathrm{AD})$ complexes iron in its core to produce the insoluble, unreactive compound ferrihydrate.

\section{CONCLUSION}

The fact that oxidative stress plays an important role in $\mathrm{AD}$ pathogenesis is apparent, given all the evidence the research has recently provided. Markers of oxidative damage including heme oxygenase-1 (HO-1) and $8 \mathrm{OHG}$ are increased in the AD brain as compared with controls. In addition, NFTs and senile plaques are altered in ways characteristic of oxidative damage including AGE-modification, protein cross-linking and carbonyl- and acyl-modification. Although the source of the shift in oxidative homeostasis is still unclear, current evidence points to changes in the balance of redox transition metals, especially iron and copper. Both $\mathrm{Fe}$ and $\mathrm{Cu}$ are present at significantly elevated levels in $\mathrm{AD}$ neuropil, and detection of redox activity in the $\mathrm{AD}$ brain can be attenuated by chelators of these key metals. It has also been demonstrated that many of the proteins that are important in their regulation, including ferritin and ceruloplasmin, show altered expression in $\mathrm{AD}$ and other neurodegenerative disorders. These changes could be responsible for the oxidative imbalance or may represent an attempted antioxidant response by affected cells.
However, the key aspect of the degree of cognitive decline in neurodegenerative disease is selective neuronal death. Our findings that neurons displaying protein damage indicate an oxidative stress response is still in need of a mechanism that links damage and death. Determining the relative contribution of sugar- or lipid-adduction reactions as well as direct side-chain oxidation to the properties of abnormal inclusions will provide insight into the mechanistic link, but also into rational therapeutic protocols for each disease, for example, using water- versus lipid-soluble antioxidants or free-radical versus carbonyl scavengers. We are encouraged by the preliminary epidemiological and clinical studies suggesting that inhibiting oxidative stress and glycation are effective in reducing the clinical manifestation of neurodegenerative diseases [19]. The stage is now set to critically examine the importance of oxidative imbalance in the pathogenesis of $\mathrm{AD}$ as antioxidants, and chelating agents take a primary role in clinical intervention of neurodegenerative diseases.

\section{ACKNOWLEDGMENTS}

This work was supported by grants from the National Institute of Aging, the Alzheimer Association, and the American Health Assistance Foundation.

\section{REFERENCES}

[1] Smith MA, Taneda S, Richey PL, et al. Advanced Maillard reaction end products are associated with Alzheimer disease pathology. Proc Natl Acad Sci USA. 1994;91(12):5710-5714.

[2] Smith MA, Harris PLR, Sayre LM, Beckman JS, Perry G. Widespread peroxynitrite-mediated damage in Alzheimer's disease. J Neurosci. 1997;17(8):2653-6537.

[3] Montine TJ, Amarnath V, Martin ME, Strittmatter WJ, Graham DG. E-4-hydroxy-2-nonenal is cytotoxic and cross-links cytoskeletal proteins in P19 neuroglial cultures. Am J Pathol. 1996;148(1):89-93.

[4] Sayre LM, Zelasko DA, Harris PL, Perry G, Salomon RG, Smith MA. 4-Hydroxynonenal-derived advanced lipid peroxidation end products are increased in Alzheimer's disease. J Neurochem. 1997;68(5):20922097.

[5] Smith CD, Carney JM, Starke-Reed PE, et al. Excess brain protein oxidation and enzyme dysfunction in normal aging and in Alzheimer disease. Proc Natl Acad Sci USA. 1991;88(23):10540-10543.

[6] Smith MA, Rudnicka-Nawrot M, Richey PL, et al. Carbonyl-related posttranslational modification of neurofilament protein in the neurofibrillary pathology of Alzheimer's disease. J Neurochem. 1995;64(6):26602666.

[7] Smith MA, Kutty RK, Richey PL, et al. Heme oxygenase1 is associated with the neurofibrillary pathology of Alzheimer's disease. Am J Pathol. 1994;145(1):42-47.

[8] Premkumar DR, Smith MA, Richey PL, et al. Induction of heme oxygenase- 1 mRNA and protein in neocortex 
and cerebral vessels in Alzheimer's disease. $J \mathrm{Neu}$ rochem. 1995;65(3):1399-1402.

[9] Takeda A, Smith MA, Avila J, et al. In Alzheimer's disease, heme oxygenase is coincident with Alz50, an epitope of $\tau$ induced by 4-hydroxy-2-nonenal modification. J Neurochem. 2000;75(3):1234-1241.

[10] Smith MA, Sayre LM, Monnier VM, Perry G. Radical AGEing in Alzheimer's disease. Trends Neurosci. 1995;18(4):172-176.

[11] Gou JP, Eyer J, Leterrier JF. Progressive hyperphosphorylation of neurofilament heavy subunits with aging: possible involvement in the mechanism of neurofilament accumulation. Biochem Biophys Res Commun. 1995;215(1):368-376.

[12] Friguet B, Stadtman ER, Szweda LI. Modification of glucose-6-phosphate dehydrogenase by 4-hydroxy2-nonenal. Formation of cross-linked protein that inhibits the multicatalytic protease. J Biol Chem. 1994;269(34):21639-21643.

[13] Nunomura A, Perry G, Pappolla MA, et al. RNA oxidation is a prominent feature of vulnerable neurons in Alzheimer's disease. J Neurosci. 1999;19(6):1959-1964.

[14] Hensley K, Carney JM, Mattson MP, et al. A model for $\beta$-amyloid aggregation and neurotoxicity based on free radical generation by the peptide: relevance to Alzheimer disease. Proc Natl Acad Sci USA. 1994;91(8):3270-3274.

[15] Odetti P, Angelini G, Dapino D, et al. Early glycoxidation damage in brains from Down's syndrome. Biochem Biophys Res Commun. 1998;243(3):849-851.

[16] Lovell MA, Robertson JD, Teesdale WJ, Campbell JL, Markesbery WR. Copper, iron and zinc in Alzheimer's disease senile plaques. J Neurol Sci. 1998;158(1):47-52.

[17] Perez M, Valpuesta JM, de Garcini EM, et al. Ferritin is associated with the aberrant tau filaments present in progressive supranuclear palsy. Am J Pathol. 1998;152(6):1531-1539.

[18] Smith MA, Harris PLR, Sayre LM, Perry G. Iron accumulation in Alzheimer disease is a source of redox-generated free radicals. Proc Natl Acad Sci USA. 1997;94(18):9866-9868.

[19] Munch G, Schinzel R, Loske C, et al. Alzheimer's disease-synergistic effects of glucose deficit, oxidative stress and advanced glycation endproducts. $J$ Neural Transm. 1998;105(4-5):439-461.

* Corresponding author.

E-mail: mas21@po.cwru.edu

Fax: +1 216368 8964; Tel: +1 2163683670 

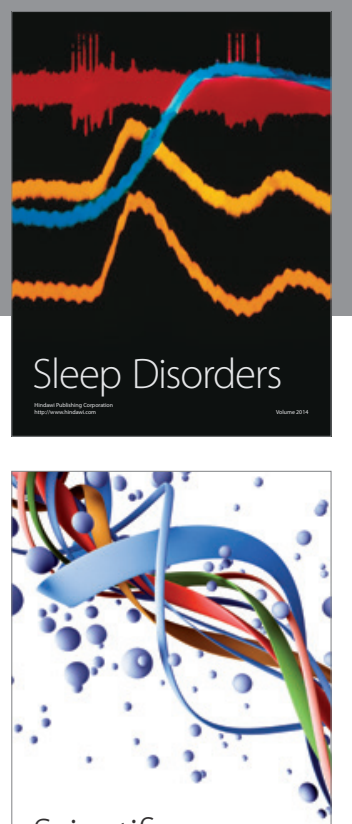

Scientifica
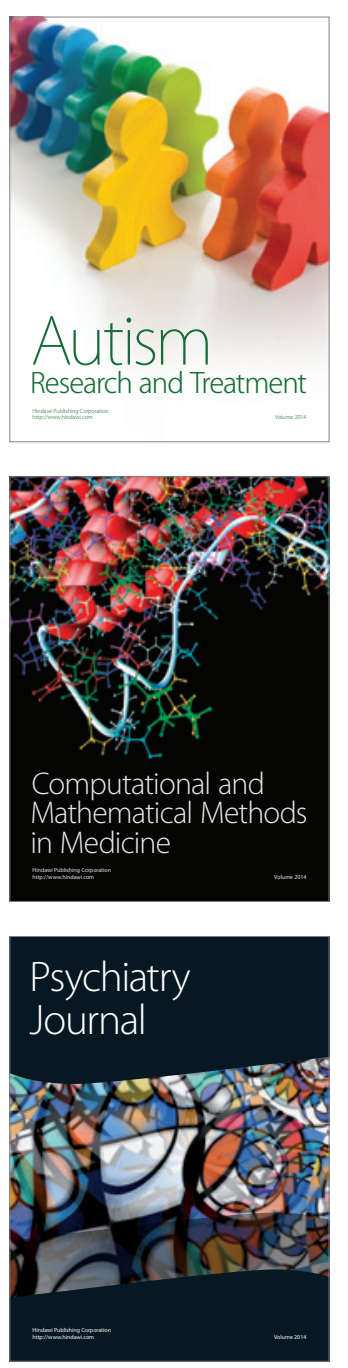
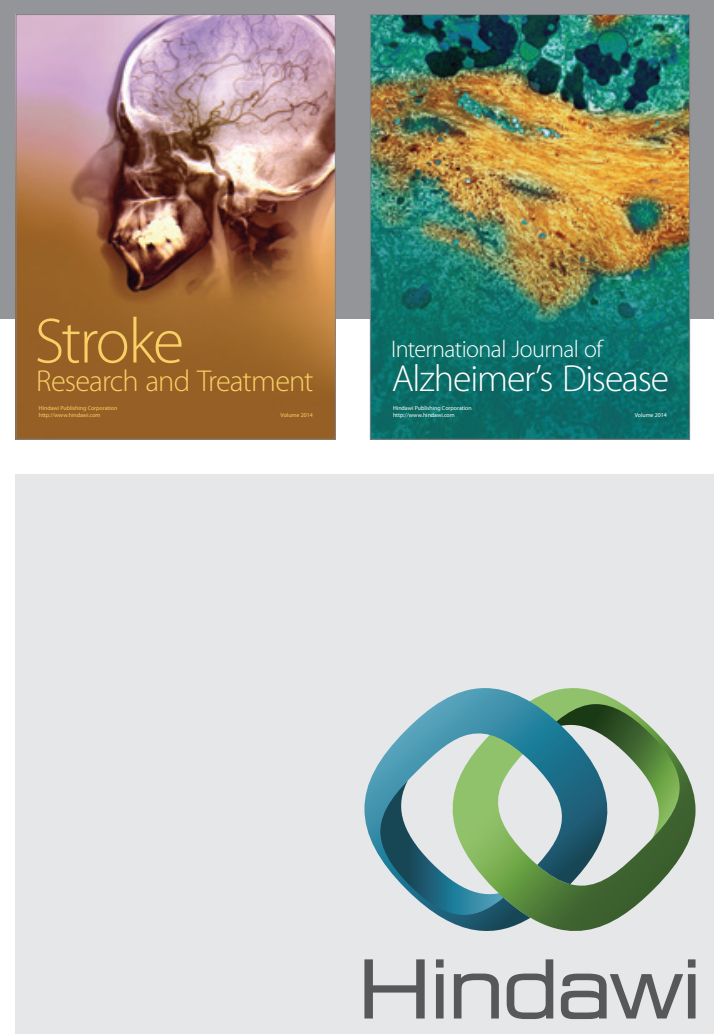

Submit your manuscripts at

http://www.hindawi.com
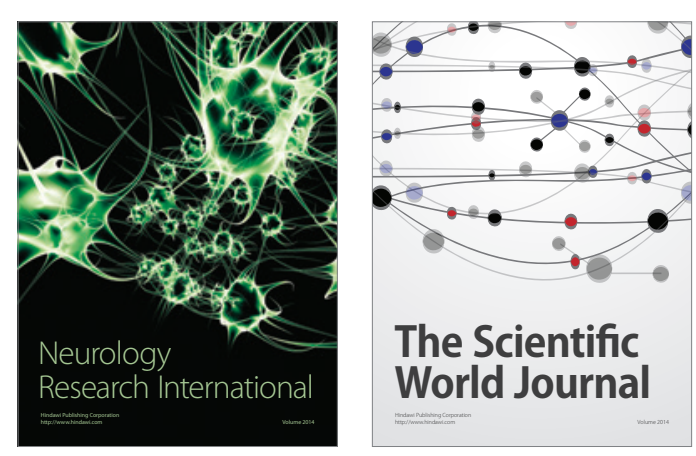

The Scientific World Journal

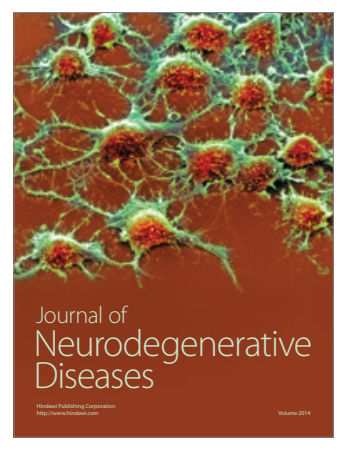

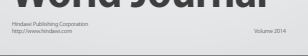

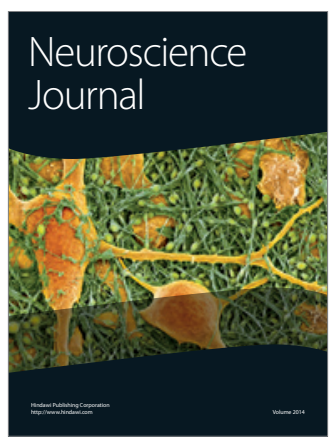

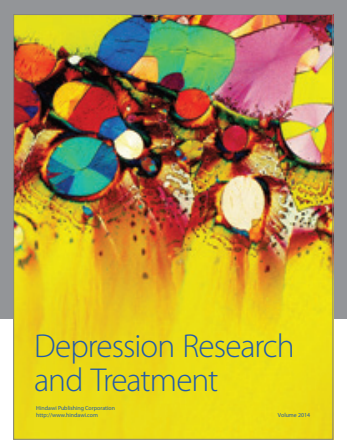
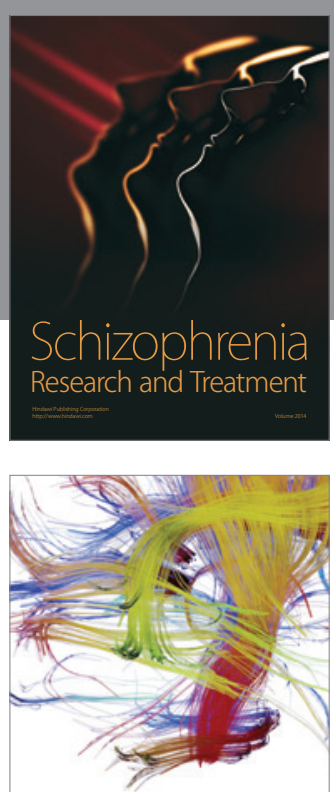

Brain Science

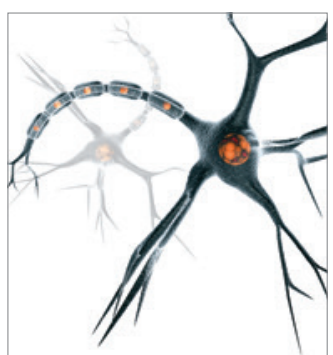

Neural Plasticity
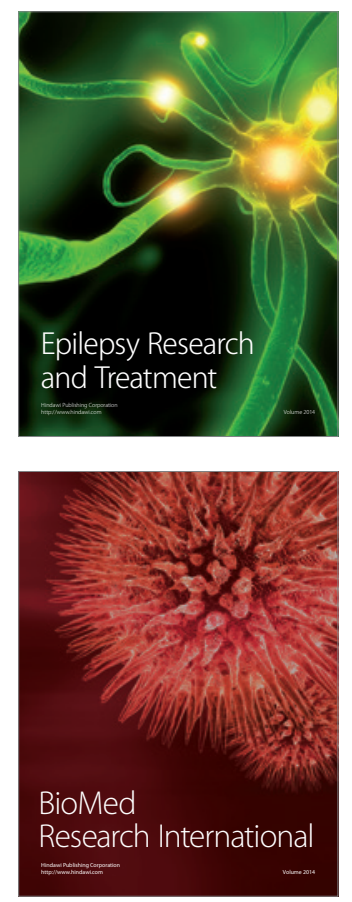

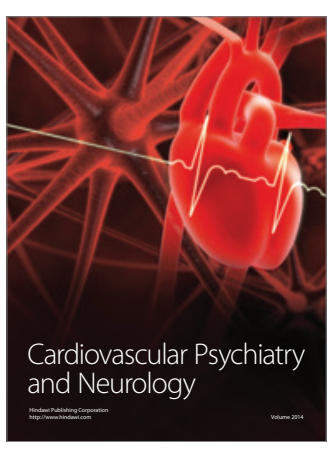

Parkinson's

Disease
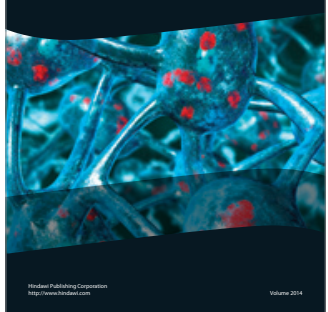